\title{
Co-Registered Application of Matrix Assisted Laser Desorption/Ionization Mass Spectrometry and Time-of-Flight Secondary Ion Mass Spectrometry Images for Visualizing Signaling Molecules
}

Matthias Lorenz ${ }^{1,2^{*}}$, Steven T. King, ${ }^{1}$ Nikolay Borodinov, ${ }^{1}$ Chad A. Steed, ${ }^{3}$ Junghoon Chae, ${ }^{3}$ Anton V. Ievlev $^{2}$ and Olga S. Ovchinnikova ${ }^{1}$

1. Oak Ridge National Laboratory, Center for Nanophase Materials Sciences, Oak Ridge, TN, USA.

2. University of Tennessee, Knoxville, Bredesen Center, Knoxville, TN, USA.

3. Oak Ridge National Laboratory, Computer Science and Mathematics Division, Oak Ridge, TN, USA.

* Corresponding author: lorenzm@ornl.gov

Matrix Assisted Laser Desorption/Ionization (MALDI) is a commonly used technique for the chemical imaging of biological tissue material with mass spectrometry (MS) because of its capability to desorb and ionize large organic molecules with a relatively low degree of fragmentation. The range of accessible analytes include heavy molecular species such as peptides and proteins with intact molecular ions observable in many cases [1]. Time-of-Flight (ToF) Secondary Ion Mass Spectrometry (SIMS) is another mass spectrometry imaging (MSI) technique that can achieve a particularly high spatial resolution below $100 \mathrm{~nm}$ [2], and that further enables three-dimensional applications by the use of sputter depth profiling. However, its analytical information is limited to smaller organic molecules and elemental species due to the decrease of ion yields with molecular weight and the significant fragmentation of molecular bonds and the loss of structural information.

MS based surface analysis techniques are inherently destructive as they necessitate the extraction of a specimen from the sample for analysis. However, the minimal volume of this specimen differs considerably for ToF-SIMS and MALDI-ToF-MS, as is also reflected in their respective achievable spatial resolution in chemical imaging $(\sim 25 \mu \mathrm{m}$ vs. $<100 \mathrm{~nm})$. This difference can be used to justify the assumption of a virtually non-destructive nature of the ToF-SIMS analysis and the development of a workflow comprising the sequential application of chemical imaging by ToF-SIMS followed by MALDI-ToF-MS on the exact same sample. In this work, we demonstrate how the multimodal combination of MALDI-ToF-MS and ToF-SIMS techniques on the same sample can be used to create a co-registered chemical imaging data set that supplements the limited molecular structural information from ToF-SIMS imaging by MALDI mass spectral information. The result is a high spatial resolution co-registered chemical imaging data set with the chemical complexity of MALDI mass spectra. We showcase our data analytics workflow for the visualization of signaling molecules in animal brain tissue thin sections.

Fresh frozen $10 \mu \mathrm{m}$ thick coronal sections of mouse brain tissue, mounted on indium tin oxide (ITO) coated microscope glass slides (Bruker Daltonics, Bremen, Germany) were purchased from Zyagen (San Diego, CA). Samples were shipped on dry ice and stored at $-80{ }^{\circ} \mathrm{C}$, thawed and desiccated under vacuum and ready for analysis by SIMS within $2 \mathrm{~h}$. MALDI analysis was performed within $24 \mathrm{~h}$. We utilized a TOF.SIMS V (IONTOF, Münster, Germany) and an Autoflex Speed (Bruker Daltonics) MALDI MS. $\alpha$-Cyano-4-hydroxycinnamic acid ( $\alpha$-CHCA) was obtained from Sigma-Aldrich (St. Louis, MO). Acetonitrile, methanol, and water were purchased from Fisher Scientific (Hampton, NH). In-house developed scripts were written using the Anaconda Python distribution (Anaconda, Austin, TX) and available open libraries OpenCV [3] and pyimzML [4] Scikit-learn [5]. The open source 
CrossVis [6] visual analytics tool was used for the visualization of multivariate data.

A fresh frozen mouse brain tissue section was thawed, desiccated and chemically imaged with ToFSIMS using a $\mathrm{Bi}_{3}{ }^{+}$liquid metal ion source (LMIS) with $1 \mu \mathrm{m}$ spatial resolution in stage scan mode with $\sim 15 \times 30$ patches of $300 \mu \mathrm{m}$ side length. Three fiduciary markers of $250 \mu \mathrm{m} \times 250 \mu \mathrm{m}$ size were etched at the corners of the tissue section following the ToF-SIMS imaging experiment using the $\mathrm{Bi}_{3}{ }^{+}$primary ion beam that was also used for analysis, but in DC mode to induce molecular damage in the confined regions that can be seen in the MALDI spectra on the tissue material. A uniform layer of $\alpha$-CHCA was applied by spray coating from acetonitrile/water solution to serve as MALDI matrix, and the tissue section imaged with MALDI-ToF MSI with $50 \mu \mathrm{m}$ spatial resolution and $0-5 \mathrm{kDa} \mathrm{m} / \mathrm{z}$ range. A second tissue thin section present on the same microscope slide was treated and imaged using identical conditions. It had not been imaged during the prior ToF-SIMS analysis and served as a control showing that the interaction with the $\mathrm{Bi}_{3}{ }^{+}$primary ion beam did not induce any chemical change of the biological material visible on the scale of the lower spatial resolution of MALDI MSI. ToF-SIMS and MALDIToF-MS MSI data sets were exported as GRD and imzML formats, respectively. In-house developed scripts based on Python were used to read the exported data sets and convert them into a common data structure that was stored in HDF file format for further data handling. The OpenCV library was used to automatically identify fiduciary markers in the MALDI MSI data set, which were used to calculate a transformation matrix based on the marker's spatial coordinates in the ToF-SIMS MSI data.

The merged data set facilitates the application of various data analytics tools, including principal component analysis (PCA) and non-negative matrix factorization (NMF). We use the CrossVis data visualization software to study and illustrate cross-correlation between signals in the merged data set. In this presentation, we show results from data mining efforts on the combined MSI datasets from ToFSIMS and MALDI-ToF-MS with a focus on signaling molecules in mouse brain tissue and use the data to discuss and illustrate the potential of the novel multi-modal approach as compared to data obtained from the individual MSI techniques [7].

\section{References:}

[1] PJ Todd et al., J. Mass Spectrom. 36 (2001), p. 355.

[2] F Kollmer et al., Surf. Interface Anal. 45 (2013), p. 312.

[3] OpenCV library, https://opencv.org/ (accessed February 22, 2019).

[4] alexandrovteam/pyimzML, https://github.com/alexandrovteam/pyimzML (accessed February 22, 2019).

[5] ORNL/CrossVis, https://github.com/ORNL/CrossVis (accessed February 22, 2019).

[6] scikit-learn, https://scikit-learn.org/ (accessed February 22, 2019).

[7] This research was conducted at the Center for Nanophase Materials Sciences, which is a DOE Office of Science User Facility, and using instrumentation within ORNL's Materials Characterization Core provided by UT-Battelle, LLC under Contract No. DE-AC05-00OR22725 with the U.S. Department of Energy. The research was sponsored in part by the Laboratory Directed Research and Development Program of Oak Ridge National Laboratory, managed by UT-Battelle, LLC, for the U.S. Department of Energy, and in part by an appointment to the Oak Ridge National Laboratory HERE Program, sponsored by the U.S. Department of Energy and administered by the Oak Ridge Institute for Science and Education. 